\title{
An Improved POD Technique for the Optimization of MSF Processes
}

\author{
R. Melli*, E.Sciubba, C.Toro, A. Zoli-Porroni \\ Department of Mechanical \& Aerospace Engineering \\ University of Roma 1 "La Sapienza" \\ E-mail: roberto.melli@uniroma1.it
}

\begin{abstract}
This paper discusses a general procedure leading to the process optimization of Multiple Flashing Desalination processes (MSF). The optimal configuration is attained via a novel inverse-design approach that uses the global exergy efficiency as objective function.
\end{abstract}

A fundamental methodological novelty of the proposed procedure is that it does not require the generation of a complete simulated set of results at each iteration step of the optimisation, because the objective function is computed by a functional extrapolation based on the Proper Orthogonal Decomposition (POD) method. With this method, the (often excessively taxing) computational cost for repeated numerical process simulations of incrementally different configurations is substantially reduced by replacing much of it by easy-to-perform matrix operations: a certain (small) number of initial process simulations is used only to calculate the basis of the POD interpolation and to validate (i.e., extend) the results.

As the accuracy of a POD expansion critically depends on the allowable number of initial simulations (the "snapshots"), the computational intensity of our methodology is certainly not negligible: but, as successfully demonstrated in the paper for a strongly simplified but realistic MSF process design problem, the idea that, given a certain number of necessary initial process simulations, additional full simulations are performed only in the "right direction" indicated by the gradient of the objective function in the solution space, leads to a successful strategy at a substantially reduced number of simulations. This "economy" with respect to other classical "optimization" methods is basically due to the capability of the POD procedure to identify the most important "modes" in the functional expansion of the vector basis consisting of a subset of the design parameters used in the evaluation of the objective function.

\section{Keywords: MSF desalination; proper orthogonal decomposition; optimization.}

\section{Introduction}

Industrial-size desalination of sea (or brackish) water has been routinely practiced for over 70 years and is a wellestablished source of water supply in several countries, most noticeably in the Middle East. It has evolved into a technically and economically feasible technology to produce large quantities of water of excellent quality from two or three different types of desalination processes (multiple flash, MSF; multiple effect, MED; reverse osmosis, RO). At the current state of the art, the challenge consists in producing desalinated water of suitable quality for different uses (industrial, domestic, agricultural) and for relatively large communities, at affordable cost.

A rough market segmentation across technologies has emerged in this strongly competitive market: for small size plants, RO and MED processes are preferred; for medium sized plants, especially those producing water for industrial final users, MED and MSF processes prevail. For large plants producing water of drinkable quality, the MSF process dominates.

The MSF is a thermal seawater distillation process: the distillate is produced by flash distillation of the hot brine inlet into the stage (Figure 1). The latent heat of the produced steam is recovered in an internal heat exchanger to heat the brine. This process is repeated across several stages: the higher the number of stages, the lower the specific energy required to produce $1 \mathrm{~m}^{3}$ of fresh water. These plants are generally realized in units of 18-24 stages, and due to economic and thermodynamic reasons, they are usually combined with a power plant operating in cogeneration mode: the MSF is fed by low-T, low-p steam $\left(140^{\circ} \mathrm{C}\right.$ and 4 bar being the standard). Standalone configurations for an MSF desalination plant are sometimes employed for small size units where low installation costs are mandatory, but in this range of applications the MSF is strongly challenged by modern RO units. As the MSF desalination plant requires low temperature heat, it is optimally suited for cogenerating applications, and several successful configurations exist that integrate the cogeneration plant with an auxiliary boiler to cover possible peaks of water production without displacing the main plant from its optimal operating point.

Fig. 2 shows how a MSF plant can be combined with a steam power plant, of which it constitutes a bottoming subunit. It is possible to identify three main infrastructures in a MSF desalination plant:

1) The power section, where both steam and electricity are produced. The economics of the system is substantially improved if the MSF plant is combined with a power plant in such a way that the steam discharged from the back-pressure turbine (at a temperature of about $140^{\circ} \mathrm{C}$ ) feeds the desalination 


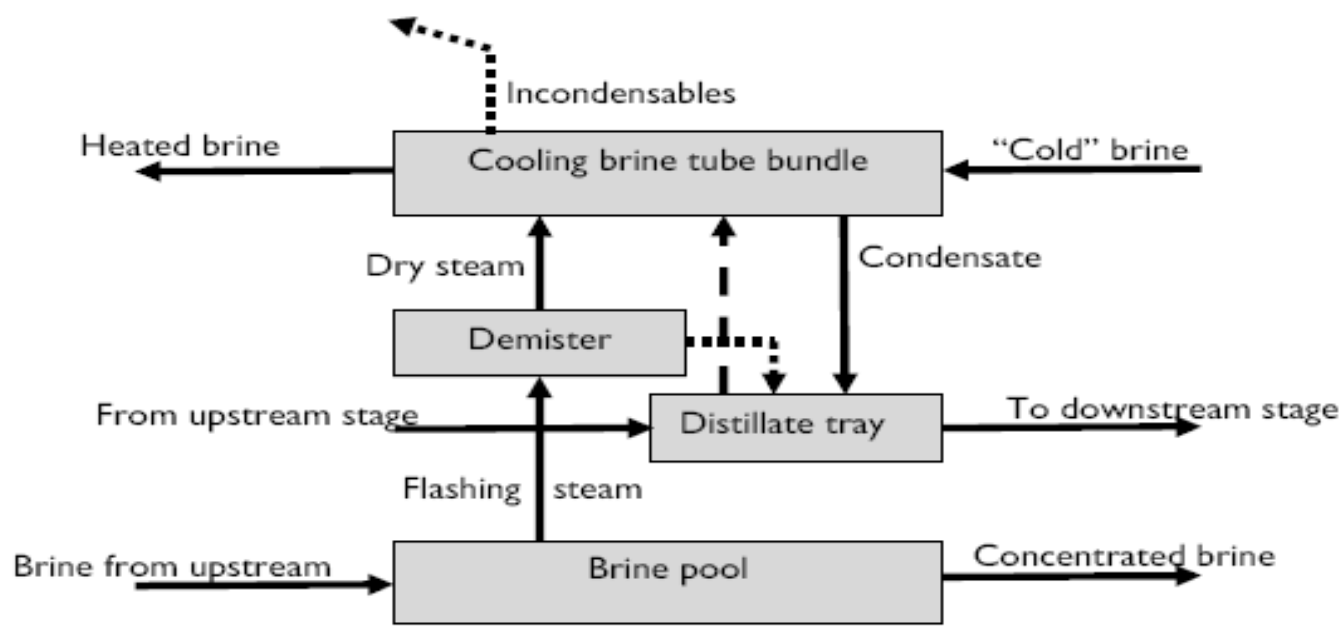

Figure 1. Simplified scheme of single MSF stage. (Dashed and dotted lines represent streams usually neglected in the energy balance).

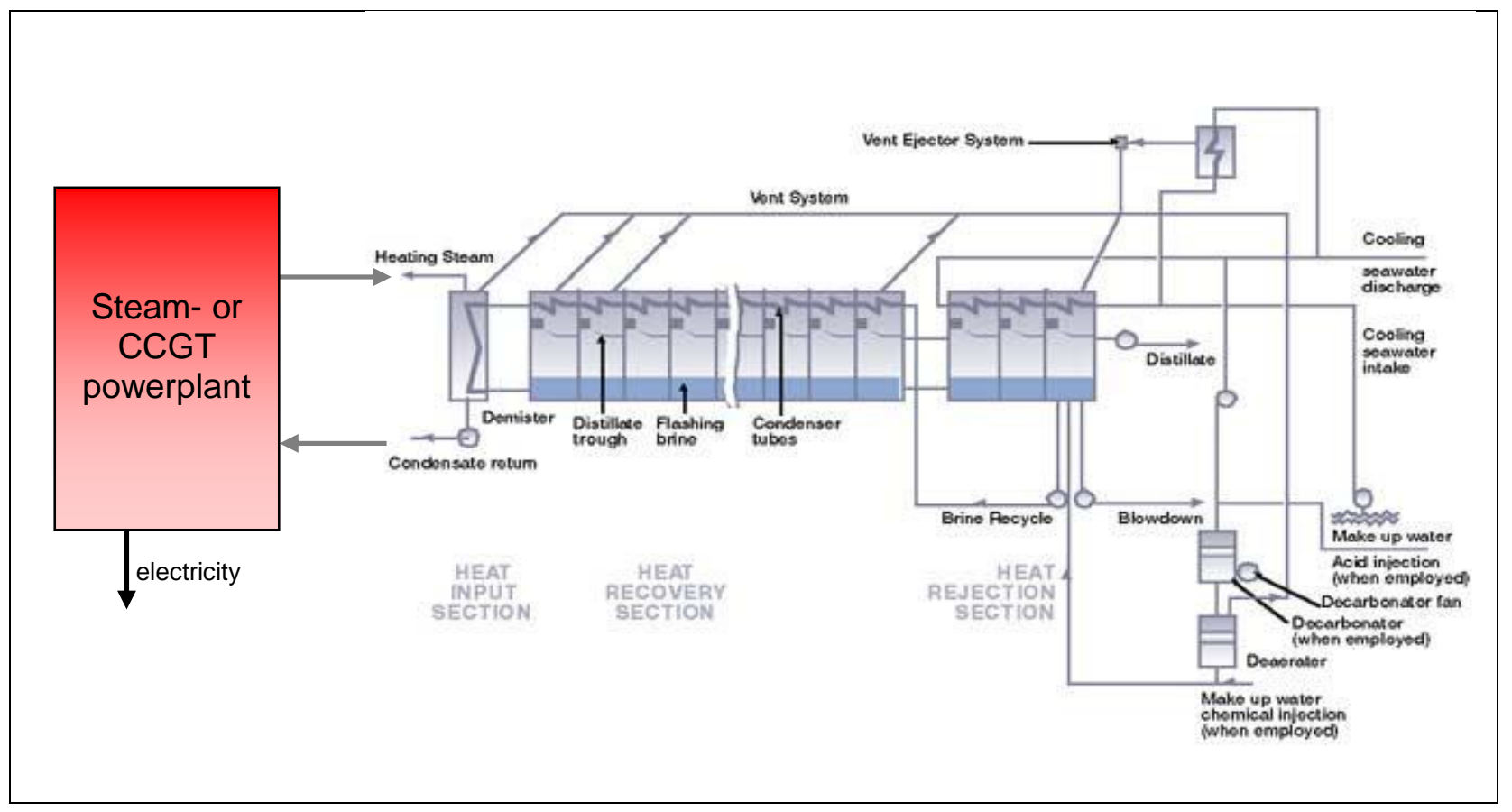

Figure 2. General layout of a large MSF plant.

plant. More recently, there are examples of MSF plants bottoming CCGT (combined cycle gas turbine). This allows for a substantial reduction of the product (distillate) cost and makes the MSF technology the only practically convenient solution for large units.

2) The MSF plant proper, where the distillate production occurs.

3) The intake seawater structure, necessary for correctly interfacing the plant with the environment.

The seawater (or brackish water, depending on the installation) is pumped into the Heat Rejection Section, usually consisting of 3-4 stages, in which it absorbs the latent heat of evaporation of the relatively cold brine and produces a relatively small amount of distillate. The heated seawater is then discharged into the sea at a temperature of about $40^{\circ} \mathrm{C}$ (the exact value depending on local environmental conditions). The highly concentrated brine ejected from the last (lowest $T$, lowest $\mathrm{p}$ ) stage -the rightmost in the figure- is partially recycled in the upstream stages and partially discharged into the sea (blow-down). The recycled brine is pumped through the stages that constitute the Heat Recovery Section (which number from 15 to 20 , depending on the plant configuration) where it flows in counterflow with the evaporating high-temperature brine coming from the Heat Input Section (a steam-to-brine heat exchanger, leftmost in the figure). At each stage, the brine absorbs the latent heat of condensation of the flashing brine in the bottom "pool", and correspondingly increases its temperature. The hot brine exiting the heat exchanger also called for obvious reasons the brine heater- at $120-135^{\circ}$ (this is called the top brine temperature, TBT) enters the first stage, which is maintained at a lower pressure than the saturation pressure corresponding to the temperature in the bottom pool: as a result, a portion of the brine evaporates, and the brine from the pool of stage 1 flows into the second stage with an increased salt concentration. The second stage 
is in turn kept at a lower pressure than that necessary for equilibrium, and the flashing process is repeated.

The vapor produced in the bottom pool of each stage flows upwards by gravity, and passes through a demister where it attains saturated conditions. Then, it condenses on the upper tube bank through which the cooling brine flows in crosscurrent, and drips on a specially shaped tray that channels it to the following stage (at lower pressure) where it mixes with the distillate generated there, to continue the process. This apparently tortuous scheme is designed to maximize the heat recovery in a global sense, i.e., to make optimal use of the heat supplied at high $\mathrm{T}$ by the steam in the brine heater and cascaded downstream.

The design of MSF plants is not an easy task, and several practical design "tricks" are necessary to ensure steady operation with a satisfactory degree of efficiency. As a consequence, ample use is made of specifically construed simulation tools to study possible process improvements. We will not delve into the details here: it suffices to say that the most taxing problems are caused by the scaling of the hot section (the brine flows through steel tubes that are prone to damage by deposits of magnesium, calcium and potassium salts by force present in seawater) and by the need of attaining a satisfactory compromise between the necessity of "sealing" each stage from its immediate neighbours and the intrinsically non-equilibrium conditions that in reality reign inside of the evaporating pool.

Since MSF desalination is a successful technology, its business is growing and the ensuing harsher competition calls for new analysis tools capable of integrating both the technical and economic aspects of the problem. A detailed analysis of the technical problems allows for the introduction of technological "fixes" aimed at avoiding operational problems, extending the time between repairs and de-scaling, and improving the overall efficiency. In last analysis, this is reflected in a reduction of the operating cost and, as a consequence, of the net cost of the product (the distillate).

An essential design task is the identification of the "optimal" stage arrangement, given by the number of stages in the Heat Recovery Section, the top brine temperature, and the operating temperature and pressure of each stage. Scope of the present paper is to demonstrate a new method for this optimization, based on an ad hoc application of a mathematical procedure called Proper Orthogonal Decomposition (POD). A correct implementation of this method enables the designer to quickly inspect the range of possible solutions (the design space) and to find with a reasonable use of computational resources the "optimal" configuration under a pre-assigned set of constraints (ElSayed, 2003) and (El-Nashar, El-Sayed, 2002).

No cost considerations have been included in the present study. A POD-based thermo-economic optimization is though possible, and will be the topic of a follow-up paper.

The next section contains a brief description of the process simulator. Section 3 provides the background of the POD method and illustrates the optimization procedure. Section 4 presents and discusses the results.

\section{MSF Process Simulation}

CAMEL-Pro ${ }^{\mathrm{TM}}$ (Italian acronym for "Modular Calculation by Elements") is an innovative modular simulation code developed and perfected over a number of years in our Process Simulation Lab. The code's task is the simulation of energy conversion systems, and in particular, thermal power plants. The main objective of the implementation of an in-house simulator was that of providing an inspectable, expandable, powerful and yet user-friendly computational tool for the analysis of power plants according to specific criteria and characterized by a substantially greater versatility of analysis with respect to similar commercial codes. Most of MSF simulators in fact do not describe in detail the processes internal to the individual stage, but treat the overall plant (or the stage) in a "lumped" fashion, aggregating some or all flashing stages in one macro-unit and not accounting for internal recycles etc. The MSF stage model has been developed in CAMELPro $^{\mathrm{TM}}$ in such a way as to allow the user to simulate any kind of MSF desalination process, regardless of its configuration, size, number of stages, and operative conditions.

CAMEL-Pro ${ }^{\mathrm{TM}}$ (www.turbomachinery.it, 2008) is written in $\mathrm{C}++$ and $\mathrm{C \#}$, is based on a completely and genuinely object-oriented approach, and is equipped with a user-friendly graphical interface that allows for the simulation and analysis of energy conversion processes of whatever configuration. The system is represented as a network of components connected by material and energy streams; each component being characterized by a set of equations describing the thermodynamic changes imposed on the streams; in mathematical terms, this equation system is not closed, and, therefore, needs of a proper number of boundary conditions in terms of known flow parameters. In practical terms, this means that the computed solution depends on both the plant configuration and on the assigned boundary conditions.

An optimized iterative Newton-Raphson algorithm is used to solve the global equation system. The main feature of CAMEL-Pro ${ }^{\mathrm{TM}}$ is in fact its modularity that enables users to expand the code by adding new components or by modifying the model of the existing ones: we exploited these capabilities to introduce the proper process equations for the MSF model. The analysis is based on energy and mass balance criteria and takes into account specific features that characterize the thermodynamic phenomenology in the stage as well as the thermodynamic properties of each stream.

CAMEL-Pro $^{\text {TM }}$ is equipped with a library of thermodynamic properties for the calculation of thermophysical properties of fluids. Models for the calculation of thermodynamic and transport properties of water and steam are implemented according to the IAPWS 2003. CAMEL has proved itself as a useful tool for both designing a new plant and analyzing the behaviour of an existing one. The code is also an important tool to simulate the off-design of an existing plant, and is of invaluable assistance for designers facing the task of developing a plant management strategy.

The MSF Stage Model in CAMEL (Figure 3) is divided into three sub sections:

1. Heat recovery,

2. Brine Pool

3. Condensate tray. 


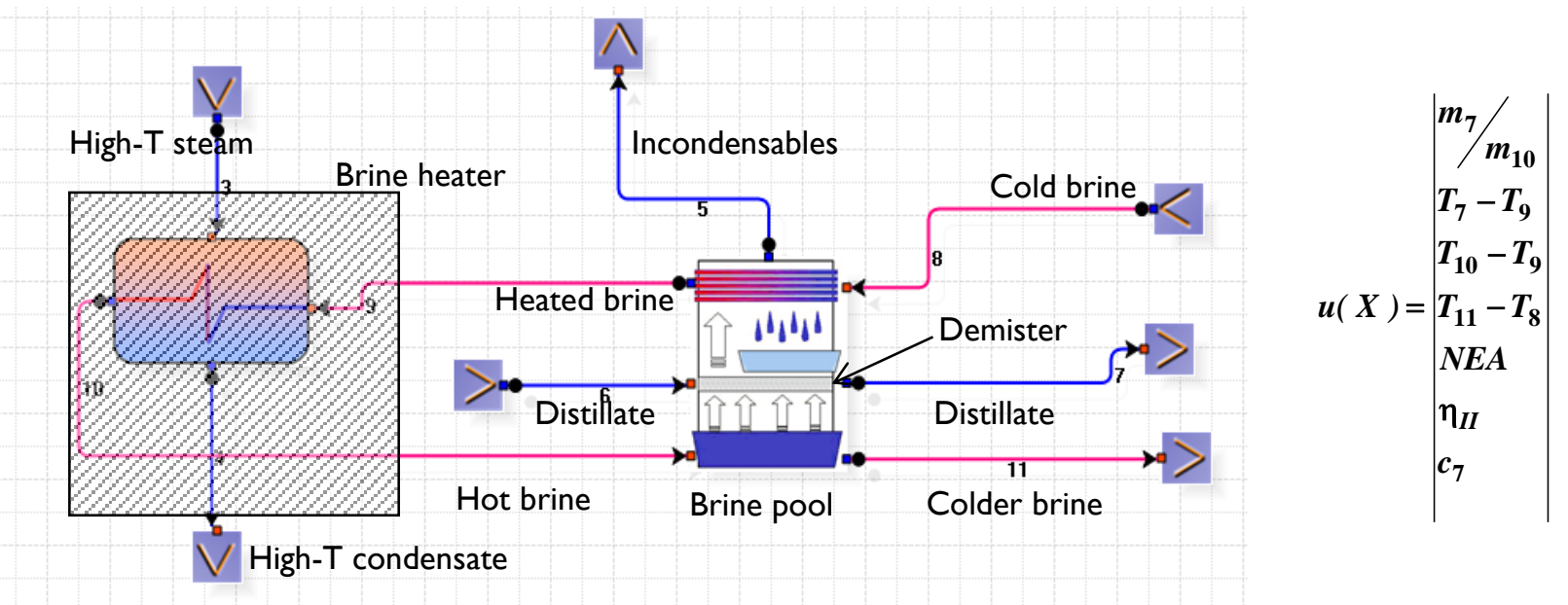

Figure 3. The MSF Stage Model in CAMEL and its snapshot. Legend: (3) hot steam; (4) cold steam; (5) incondensables; (6) distillate from upstream stage; (7) distillate to downstream stage; (8) colder brine from downstream stage; (9) heated brine to upstream stage; (10) concentrated brine from upstream brine pool); (11) concentrated brine to downstream brine pool. First stage shown for clarity.

\subsection{CAMEL MSF Model Description}

We have seen above that the MSF process with brine recirculation consists of a series of stages in which flashing evaporation takes place from a brine flowing through the bottom of the stage. The vapor released in the flashing process passes through the demisters located just above the free surface to remove brine droplets and then condenses on cooling tubes at the top of the stage. Seawater (in the Heat Release Section) or brine (in the Heat Recovery Section) flowing through the tubes is heated by the latent heat of the condensing vapor, experiencing a temperature rise somewhat smaller than the temperature drop in the bottom flashing. The net heat to maintain the whole process is of course supplied by the steam condensing in the brine heater (Heat Input Section), that provides the thermal forcing needed for evaporation and condensation processes in the stages. Heat is rejected to the environment from the lowest temperature group of stages, which are directly cooled by seawater flowing through the tubes. In CAMEL, the single stage is "unaware" of what is happening in the up- and downstream stages: its calculation is based only on the local mass- and energy balances, and its connection with the other stages is forced by a proper assembling of the global equation system. This is obtained by proper relabeling of the local variables during the assembly phase, so that, for instance, the brine exiting from stage number $i$ is assigned the same label as that entering stage number $i+1$ (stages are numbered starting from the brine heater and proceeding downstream). More details about the code are given in (ElSayed, 2003).

The MSF desalination process has been extensively studied from a thermodynamic point of view, to better analyze design criteria and investigate how the main parameters can affect the process. If the steam temperature is an external constraint and not a variable of the project, it imposes a limit on the number of stages. An analysis of a real plant (El-Nashar, El-Sayed, 2002) has shown that it is possible to increase the number of stages with a fixed steam temperature, to fully exploit the physical limit posed by the TBT. In that analysis, the number of stages (42), was in fact the physical limit imposed by the TBT.

Another important result obtained in a series of simulations of real processes (El-Nashar, El-Sayed, 2002) that the distillate production is not affected by the number of stages, but only by the total heat surface of the recovery section. In designing a new plant, the total heat surface can be thus fixed as soon as the request of fresh water is known. The number of stages is then calculated on the basis of the physical limitations for the heat exchanges (the stage $\Delta \mathrm{T}$ ) and of an economic analysis that includes the steam cost variation with temperature and pressure.

Since MSF plants are bottom units of a cogenerated steam cycle, an exergy analysis, also performed by CAMEL, allows to optimize the allocation of the available energy resources between the two products (distillate and electricity). The exergy analysis has proved itself as a valid tool also in identifying different strategies to evaluate offdesign conditions and to optimise the result under site dependent constraints.

\section{The Proper Orthogonal Decomposition Method}

The Proper Orthogonal Decomposition method (POD) has its roots in statistical analysis and produces a set of empirical eigenfunctions that describes the dynamics of a given problem. Several versions of POD have been published over the years, under different names, like Principal Component Analysis (PCA), Karhunen-Loève Decomposition (KLD), or Singular Value Decomposition (SVD), (Karhunen, 1946). We shall follow here in essence Sirovich' description (Sirovich, 1987): he nicknamed POD "the method of snapshots", and recommended it as an efficient way for determining the coefficients of a "proper" (in a sense discussed here below) eigenfunctions expansion for large problems for which a set of initial data is known not functionally but as a set of "experimental" data points.

In essence, POD is a procedure that provides an optimal linear basis for the reconstruction of multidimensional data: its main advantage is that it allows for a substantial reduction in the order of the system under consideration. Another very useful feature is that it filters out data noise very efficiently.

In principle, the method is like any other data fitting procedure: it provides a parametric fit of a set of given data "points" (in a multidimensional space) by finding an appropriate series -and its coefficients- that converges rapidly and robustly to a satisfactorily degree of accuracy. The conceptual novelty is that POD extracts both the interpolating functions and the coefficients from the 
information already "hidden" in the data set. In addition to being optimal in a least squares sense, POD has thus also the remarkable property of using a completely data dependent modal decomposition and therefore does not need any a priori assumption about, or knowledge of, the process that generates the data. This property is advantageous when the available knowledge of the underlying process is insufficient to warrant an appropriate choice of the functional expansion basis. Running POD also helps to explore patterns in data so as to reveal "hidden" characteristics of the underlying process that generates them.

First, we provide a synthetic description of the procedure: in the second part of this section, the operational macro-instructions are provided as well.

Let us assume that a process is described by a certain number $\mathrm{N}$ of data, in the sense that every instantiation of the process can be completely described by $\mathrm{N}$ relevant independent physical quantities $(\mathrm{p}, \mathrm{T}, \mathrm{m}, \mathrm{h}, \mathrm{s}, \eta, \mathrm{x} \ldots)$ at different points on the thermodynamic process diagram). As an example, we shall use the simple Brayton cycle illustrated in Figure 4: the relevant parameters are shown in the vector at right, that -following Sirovich- we shall call "snapshot". Every snapshot, that we refer to as $\mathbf{u}(\mathbf{x})$, where $\mathbf{x}$ is the ordered vector of the $\mathrm{N}$ relevant variables, is therefore considered, according to the problem specification, a complete description of one single instantiation of the process. Since each snapshot represents, in the $\mathrm{N}$-dimensional design space, one single "experimental point", to use POD we must obtain, by numerical or physical experiments, a sufficiently abundant set of such snapshots whose numbers must be determined on a case basis.

First, the POD method considers the matrix obtained by assembling a certain number of snapshots, say $\mathbf{M}$ : in complete analogy with algebraic, polynomial or trigonometric series fitting methods, the higher $\mathrm{M}$, the more accurate the fitting, but more computationally intensive the procedure. The aim is to seek a finite dimensional representation of the function $\mathbf{u}(\mathbf{x})$ (of length $\mathrm{N}$ ) in terms of a basis $\left\{\Phi_{\mathrm{j}}(\mathbf{x})\right\}$ for $(1<\mathrm{j}<\infty)$ which allows for an approximation to the ensemble of $\mathbf{u}(\mathbf{x})$ to be constructed:
$\mathbf{U}=\boldsymbol{\Phi} \cdot \mathbf{A}$

where $\left\{\square_{\mathrm{j}}(\mathbf{x})\right\}$ are the basis functions (each column being a proper mode of the ensemble $\mathbf{U}), \mathbf{A}$ is the $(\mathrm{KxM})$ coefficient matrix of the decomposition and $\mathbf{U}$ is the NxM snapshot matrix, each column of which is a single snapshot. The analyst is of course interested in using a reduced decomposition, in which not all modes are used, but only a properly truncated subset thereof, of dimension $K$.

After having assembled the available snapshots into the matrix $\mathbf{U}$, the covariance matrix $\mathbf{D}$ is computed by premultiplying $\mathbf{U}$ by its transpose $\mathbf{U}^{\mathrm{T}}$, and the eigenvectors and the corresponding eigenvalues of $\mathbf{D}$ are extracted. The eigenvalues are first ranked and then "pruned" by discarding those that are "negligible" by some norm with respect to the others: this is achieved by employing an "energy" method, based on a linear algebra theorem that states that the energy of the $k$-th mode (square of amplitude) scales with the corresponding eigenvalue $\lambda_{k}$. The basis is accordingly truncated by first deciding the fraction of energy $\beta$ that we wish to include, and then finding the mode number $K$ that fulfils:

$$
\sum_{\mathbf{1}}^{K} \lambda_{k} \leq \beta \sum_{\mathbf{1}}^{M} \lambda_{k}
$$

Two additional theorems of linear algebra guarantee (Bialecki, Kassab., Ostrowski, 2003) that the thus truncated POD basis $\Phi_{K}$ is optimal in the sense that no other orthogonal basis carries more energy in the same number of modes and that from all possible bases the POD has the smallest entropy (smallest data noise).

Since the procedure intrinsically guarantees that the amplitude vectors of subsequent snapshots are orthogonal (non-correlated), once we have obtained a truncated expansion $\Phi_{K}(\mathrm{NxK})$, each column $\alpha_{\mathrm{K}}$ of the truncated coefficient matrix $\mathbf{A}_{\mathrm{K}}$ can be computed as:

$\alpha_{K}^{m}=\Phi_{K}^{T} \cdot u^{m}$

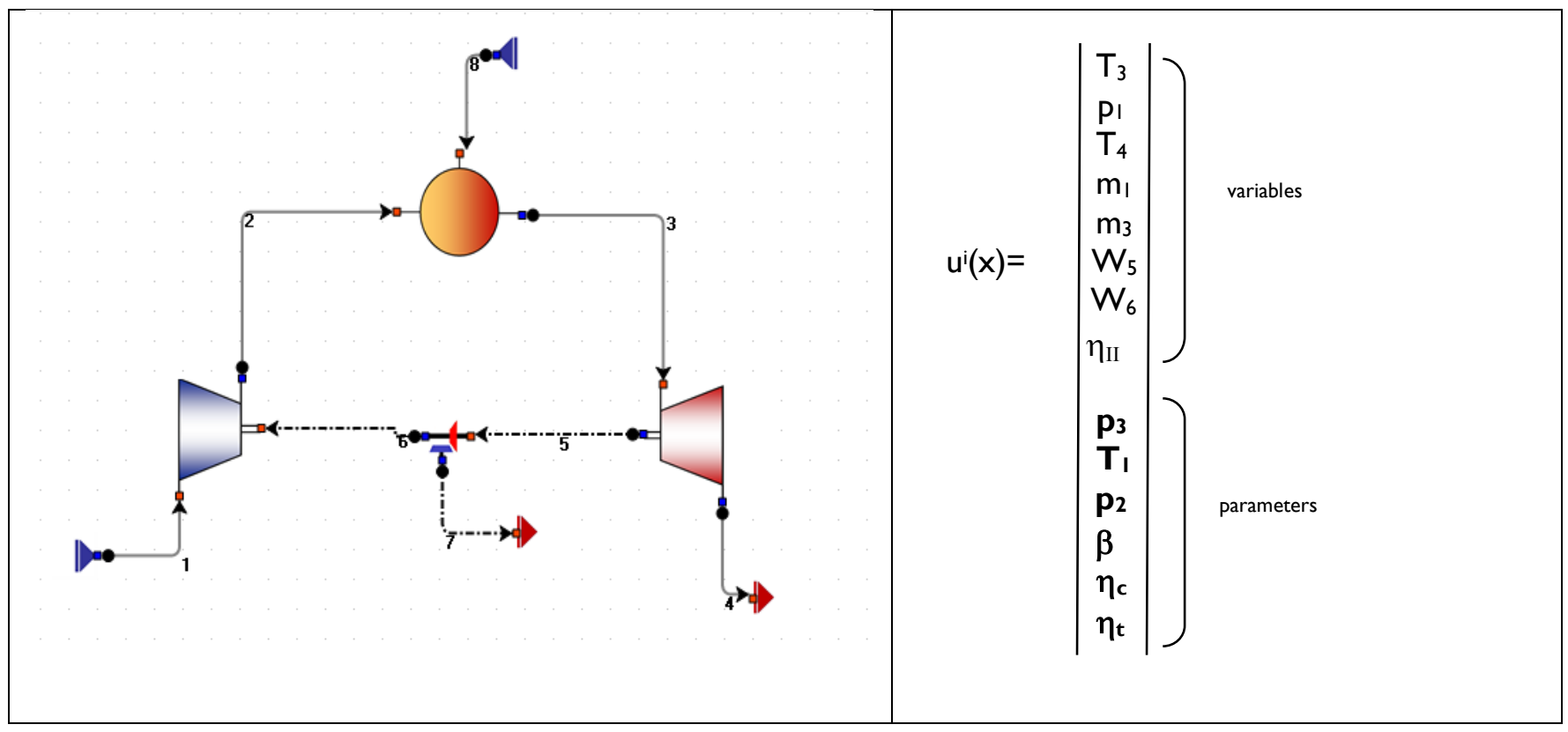

Figure 4. The simple Brayton cycle and one of its "snapshots". 
Once the proper decomposition given by equations 1 and 3 is thus known, the same expansion can be used to generate any additional "snapshot", i.e., to locate a new point in the design space corresponding to arbitrary variations of one or more of the relevant variables $\mathbf{x}$. To do this, we first assume the following form for $\boldsymbol{\alpha}_{\mathbf{K}}$ :

$$
\alpha_{K}^{m}=B \cdot f^{m}
$$

Where $f$ is a vector of length $\mathrm{K}$ representing an arbitrary norm of the derangement of the new vector $\mathbf{x}^{\prime}$ from the $\mathbf{x}^{j}$ $(j=1 \ldots M)$ contained in the original $\mathbf{U}(\mathbf{x})$ data set and $\mathbf{B}$ is an unknown (KxK) matrix. Following (Bialecki R.A., Kassab A.J., Ostrowski,, 2003) and (Ostrowski, 2006) we adopt for $f$ the so-called Radial Basis functional form. Thus, there will be $\mathrm{K}$ vectors $\mathbf{f}^{\mathrm{k}}$ defined as:

$$
f_{j}^{m}=\frac{1}{\left|\tilde{x}_{j}^{m}-x_{j}\right|+r^{2}}
$$

Where $\tilde{\boldsymbol{x}}_{j}$ is the $j$-th relevant variable in the $m$-th snapshot, $\boldsymbol{x}_{\boldsymbol{j}}$ the corresponding value in the new snapshot we wish to compute, the norm $\left|\Delta \boldsymbol{x}_{j}\right|=\sqrt{\left(\Delta \boldsymbol{x}_{j}\right)^{2}}$ and $r$ is an arbitrary smoothing factor $\left(1 / r^{2}\right.$ is an upper bound for $\left.f_{j}\right)$. The transpose of $\mathrm{B}$ is calculated as:

$$
B^{T}=\left(F^{T}\right)^{-1} A^{T}
$$

$\boldsymbol{F}(\mathrm{MxK})$ being the matrix whose columns are the $\boldsymbol{\alpha}_{\boldsymbol{K}}^{\boldsymbol{m}}$

Now, assuming that the decomposition is valid for every

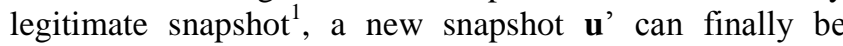
calculated as:

$$
\boldsymbol{u}^{\prime}\left(\boldsymbol{x}^{\prime}\right)=\Phi_{K} \cdot \boldsymbol{B} \cdot \boldsymbol{f}^{\prime}
$$

The entire procedure is in practice quite easily programmed in MATLAB or any other symbolic mathematic manipulator: actually, several of the above described operations are grouped in single-steps procedures that make the programming effort minimal, so that the most computationally intensive procedure is the process simulation.

\section{Results of the Optimization Procedure}

To demonstrate the performance of the POD procedure, the thermodynamic optimization of two simple processes was performed: a simple gas turbine cycle and a single stage MSF plant. These are clearly two elementary test benches, but have been chosen in such a way to allow for an immediate numerical validation.

The problem with the two case studies presented here is such that the number of streams and of the respective relevant design parameters is rather limited, which allows for a relatively small number of snapshots. Both tests are successful, in the sense that the optimal design point identified by the POD is actually the same that can be found via a numerical process simulator. When compared

I This means that the $\mathrm{N}$-tuple $\mathrm{x}_{\mathrm{i}}$ that define the new snapshot falls within some pre-established and physically feasible range in the design space with other published POD optimizations, our examples are characterized by an unusually low ratio between the number of "variables" (the design quantities that span the solution space where the optimum is sought) and that of the "parameters" (the physical quantities that identify the state of the working fluids at selected stations in the process). In real more complex cases a high ratio is known to potentially endanger the convergence of the procedure, and therefore it is to be expected that the POD can be applied with even higher confidence to more complex plants layouts.

\subsection{A Test-Case: the Simple Brayton Cycle}

The objective function to be maximized for this case is the exergy efficiency of the plant.

The selected design variables and their respective ranges are presented in Table 1.

63 combinations of the design variables were calculated via CAMEL-Pro, and 7 process parameters were extracted from each set of results:

1. The compressor inlet mass flow $\mathrm{m}_{1}$

2. The turbine inlet mass flow $m_{3}$

3. The turbine inlet temperature $\mathrm{T}_{3}$

4. The turbine outlet temperature $\mathrm{T}_{4}$

5. The turbine outlet gross power $\mathrm{W}_{5}$

6. The net available power $\mathrm{W}_{7}$

7. The exergy efficiency of the plant $\eta_{\mathrm{II}}=\mathrm{W}_{7} /\left(\mathrm{m}_{8} * \mathrm{e}_{8}\right)$

Every ordered septuple of these parameters constitutes a snapshot. After the matrix manipulation was performed, the following eigenvalues were obtained:

$\lambda_{1}=2.210^{8} ; \lambda_{2}=1.1310^{6} ; \lambda_{3}=2.610^{4} ; \lambda_{4}=910^{3} ; \lambda_{5}=3.2710^{3} ;$ $\lambda_{6}=2.1710^{3} \ldots$

of which only the first 6 were considered, since the $7^{\text {th }}$ mode is of one order of magnitude smaller and exerts a negligible influence on the expansion.

The min.cost.function routine in MatLab was used to perform the optimization, within the specified design space.

The result is an optimal set of design variables that minimize the objective function. Its accuracy may be increased by reducing the coarseness of the initial snapshot matrix $\mathbf{U}$ : but the obtained result is the most accurate optimum that can be extracted from the given collection of snapshots.

The POD locates the optimum configuration at the following design point: $\mathrm{p}_{3}=397, \mathrm{~T} 1=313 \mathrm{~K}, \mathrm{p}_{2}=405 \mathrm{kPa}, \beta=$ $4.0000, \eta_{\mathrm{C}}=1, \eta_{\mathrm{T}}=1$, for an optimal plant efficiency $\eta_{\mathrm{II}}=$ 22.9\%: as a validation, these values where inserted in the process simulator and the same efficiency was obtained in output.

\subsection{A Single-Stage MSF Process}

The procedure is obviously similar to that tested above and also in this case the objective function is the exergy efficiency of the plant. With reference to Figure 3, the selected design variables and their respective ranges are presented in Table 2 .

18 combinations of the design variables were calculated via CAMEL-Pro, and 7 process parameters were extracted from each set of results: 
Table 1. The Brayton Cycle selected design variables and their respective ranges

\begin{tabular}{|l|c|c|}
\hline \multicolumn{1}{|c|}{ Design Variable } & Symbol (Figure 4) & Range \\
\hline Compressor outlet pressure & $\mathrm{p}_{2}$ & 200 to $400[\mathrm{kPa}]$ \\
\hline Compressor inlet temperature & $\mathrm{T}_{1}$ & 298 to $313[\mathrm{~K}]$ \\
\hline Compressor efficiency & $\eta_{\mathrm{c}}$ & 0.85 to 1.00 \\
\hline Compressor & $\beta$ & 2 to 4 \\
\hline Turbine efficiency & $\eta_{\mathrm{t}}$ & 0.85 to 1.00 \\
\hline Turbine inlet pressure & $\mathrm{p}_{3}$ & 198 to $397[\mathrm{kPa}]$ \\
\hline
\end{tabular}

Table 2. The MSF selected design variables and their respective ranges

\begin{tabular}{|l|c|c|}
\hline \multicolumn{1}{|c|}{ Design Variable } & Symbol (figure 4) & Range \\
\hline Cooling brine inlet pressure & $\mathrm{P}_{8}$ & 224 to $450[\mathrm{kPa}]$ \\
\hline Cooling brine inlet temperature & $\mathrm{T}_{8}$ & 370 to $375[\mathrm{~K}]$ \\
\hline Heating brine temperature & $\mathrm{T}_{10}$ & 385 to $418[\mathrm{~K}]$ \\
\hline Pressure loss (brine tubes) & n.a. & 0.1 to $0.2[\mathrm{kPa}]$ \\
\hline Pressure loss (demister) & n.a. & 0.1 to $0.2[\mathrm{kPa}]$ \\
\hline
\end{tabular}

1. The distillate-to-brine ratio $\mathrm{m}_{7} / \mathrm{m}_{10}$,

2. The temperature difference between the distillate and the heated brine, $\left(\mathrm{T}_{7}-\mathrm{T}_{9}\right)$,

3. The temperature difference between the heating brine inlet and the cooling brine outlet, $\left(\mathrm{T}_{10}-\mathrm{T}_{9}\right)$,

4. The temperature difference between the heating brine outlet and the cooling brine outlet, $\left(\mathrm{T}_{11}-\mathrm{T}_{8}\right)$

5. The Non-Equilibrium Allowance ${ }^{2}$, NEA $\left(\mathrm{T}_{11}-\mathrm{T}_{\text {flashing }}\right.$ steam)

6. The exergy efficiency:

$$
\eta_{I I}=\frac{m_{7} e_{7}-m_{6} e_{6}+m_{8}\left(e_{9}-e_{8}\right)}{m_{10} e_{10}-m_{11} e_{11}}
$$

7. The exergy cost of the distillate:

$c_{7}=\frac{m_{3}\left(e_{3}-e_{4}\right)}{m_{7} e_{7}}$

Every ordered septuple of these parameters constitutes a snapshot. The following eigenvalues were obtained (rounded to the first two significant digits):

$\lambda_{1}=19.47 \quad 10^{3} ; \quad \lambda_{2}=2.03 \quad 10 ; \quad \lambda_{3}=0.19 ; \lambda_{4}=5.85 \quad 10^{-3} ;$ $\lambda_{5}=8.8910^{-4} ; \lambda_{6}=2.7710^{-6} \ldots$

of which only the first 5 were considered, since the $6^{\text {th }}$ mode is likely to have a negligible influence on the expansion.

The POD locates the optimum configuration at the following design point: $\mathrm{p}_{8}=441 \mathrm{kPa}, \mathrm{T}_{8}=375 \mathrm{~K}, \mathrm{~T}_{10}=418$ $\mathrm{K}, \Delta \mathrm{P}_{\text {(brine side) }}=14 \%, \Delta \mathrm{P}_{\text {(demister) }}=0 \%$.

The exergetic efficiency is 0.96 and the exergy cost of the distillate is $\mathrm{c}_{7}=15.84 \mathrm{~kJ} / \mathrm{kg}$

\section{Conclusions}

The procedure has been demonstrated to produce "correct" results. In the case of the gas turbine, the

\footnotetext{
2 This definition is used here for simplicity, and includes the so-
} called "boiling point elevation", BEP, into the NEA optimum is attained for the highest compressor and turbine efficiency, the lowest combustor pressure drop, and the highest TIT. This result is intuitive, of course, but, more importantly for our purposes, has been numerically verified: the values of the design parameters identified as "optimal" by the POD have been inserted into the process simulator and the outcome of the calculations are in exact agreement with the values "approximated" by the POD functional expansion.

For the MSF stage, the optimal design identified by the POD suggests to use the maximum allowed values for both the cooling brine and the heating temperatures, a high value of the cooling brine pressure, a rather high brine tubes pressure drop but a zero pressure drop in the demister: these indications are also intuitive for the specialist, but they prove the adequacy of the proposed procedure. Again, the POD forecast has been validated by a direct design simulation implemented in CAMEL.

These results are encouraging, and suggest to extend the application of the procedure to more complex configurations (a realistic multi-stage MSF) and to include in the calculations the equipment costs, so that a thermoeconomic optimum may be sought after and compared with the results of a standard thermo-economic optimization.

\section{Nomenclature}

$\mathrm{m}=$ mass flow rate

$\mathrm{p}=$ pressure

NEA = Non-Equilibrium Allowance

$\mathrm{T}=$ temperature

$\mathrm{W}=$ power

$\beta=$ compressor pressure ratio

$\eta_{\|}=$exergy efficiency

$\lambda=$ eigenvalues

$\eta_{\mathrm{c}}=$ compressor efficiency

$\eta_{\mathrm{T}}=$ turbine efficiency

\section{References:}

Bialecki R.A., Kassab A.J., Ostrowski Z. (2003). Application of the Proper Orthogonal Decomposition in Steady State Inverse Problems. Proceedings Inverse 
Problems Engineering Mech. IV, Masa Tanaka (ed.), Elsevier, Amsterdam-Boston, 3-12.

Rowley C.W., Colonius T., Murray R.M. (2004). Model reduction for compressible flows using $P O D$ and Galerkin projection, Physica D 189 115-129, 2004.

Chatterjee A.: An introduction to the proper orthogonal decomposition, Current Science, vol. 78,NO.7, 10 April 2000.

Colonna P., van der Stelt T.P. (2004). FluidProp: a program for the estimation of thermo physical properties of fluids, Energy Technology Section, Delft U. Techn., The Netherlands (www.FluidProp.com).

El-Sayed, Y., Aplenc, A. (1970). Application of the Thermoeconomic Approach to the Analysis and Optimization of a Vapor Compression Desalting System, J. Eng. for Power, Jan., 17-26.

El-Sayed Y., Evans R. (1970). Thermoeconomics and the Design of Heat Systems, J. Eng. for Power, Jan., 27-35.

El-Sayed Y. (2003). The Thermoeconomics of Energy Conversion, Pergamon.

El-Nashar A.M., El-Sayed Y. (2002). The Economic Feasibility of a High Efficient Gas turbine Cogeneration Option with a Hybrid MSF/RO Desalination-Thermal Power Plants, Encyclopedia of Life Support Systems (EOLSS), C08/E3-10-03-11, EOLSS Pub., Oxford UK.

Falcetta M.F., Sciubba E. (2002). A computational, modular approach to the simulation of powerplants, Heat Recovery Systems \& CHP, 15, n. 2.

Fiorini P., Sciubba E., Sommariva C. (2001). A new formulation for the non-equilibrium allowance in MSF processes, Desalination 136.

Frangopoulos C.A. (2003). Methods of Energy Optimization, Proc. Summer School "Optimisation of energy systems and processes", Gliwice (Poland), June 24-27.
IAPWS (2003). Release of the Industrial Formulation for the Thermodynamic Properties of Water and Steam, Int. Assoc. Properties Water \& Steam, Erlangen, Germany.

Grau K.( 2007). Applications of the Proper Orthogonal Decomposition Method, Internship Report CERFACS September.

Karhunen K. (1946). Über lineare methoden für wahrscheinligkeitsrechnung, (On linear methods in the calculus of probability), Ann. Ac. Sci. Fennicae, Series A1 Mathematical Physics, 37, 3-79.

Kotas T.J. (1995). The Exergy Method of Thermal Plant Analysis, Butterworths Pub.

Loeve M.M. (1955 ). Probability Theory, Van Nostrand Pub.

Graham M.D., Lane S.L., Luss D. (1993). Proper Orthogonal Analysis of Spatiotemporal Temperature Patterns, J. Phys. Chem. 1993, 889-894.

Ostrowski Z (2006). Application of Proper Orthogonal Decomposition to the Solution of Inverse Problems, Ph.D. Thesis, Silesian U. of Technology.

Panzini G., Sciubba E., Zoli-Porroni A. (2007). An application of the POD method to the entropy-based optimization of a turbine blade, Proc. ECOS'07.

Sirovich L. (1987). Turbulence and the dynamics of coherent structures, Parts I, II and III, Q. Appl. Maths XLV, 561-590.

Speigler K.S., El-Sayed Y. (1962). A Desalination Primer, Balaban Desalination Publications, 1993.

Tribus M., Evans R.: The Thermoeconomics of Seawater Conversion, UCLA Rep. n. 62-63, August.

www.turbomachinery.it (2008). CAMEL-Pro ${ }^{\mathrm{TM}}$ Users Manual, rev. 4.

Zoli Porroni A. (2004). Development and Applications of Inverse Design Techniques to an Air-cooled Gas turbine Stage, M.Eng. Thesis, U. of Roma 1. 